\title{
WELL-POSEDNESS AND DECAY PROPERTY FOR THE GENERALIZED DAMPED BOUSSINESQ EQUATION WITH DOUBLE ROTATIONAL INERTIA
}

\author{
Zaiyun Zhang*, Jianhua Huang and Mingbao Sun
}

\begin{abstract}
In this paper, we investigate the initial value problem (IVP henceforth) associated with the generalized damped Boussinesq equation with double rotational inertia

$$
\left\{\begin{array}{l}
u_{t t}+\gamma \Delta^{2} u_{t t}-a \Delta u_{t t}-2 b \Delta u_{t}-\alpha \Delta^{3} u+\beta \Delta^{2} u-\Delta u=\Delta f(u), \quad x \in \mathbf{R}^{n}, t>0, \\
u(x, 0)=u_{0}(x), \quad u_{t}(x, 0)=u_{1}(x), \quad x \in \mathbf{R}^{n} .
\end{array}\right.
$$

Based on decay estimates of solutions to the corresponding linear equation, we establish the decay estimates and the pointwise estimates by using Fourier transform. Under small condition on the initial data, we obtain the existence and asymptotic behavior of global solutions in the corresponding Sobolev spaces by time weighted norms technique and the contraction mapping principle.
\end{abstract}

\section{Introduction}

We study the Cauchy problem of the generalized damped Boussinesq equation with double rotational inertia in $n$ space dimensions

$$
\begin{aligned}
& u_{t t}+\gamma \Delta^{2} u_{t t}-a \Delta u_{t t}-2 b \Delta u_{t}-\alpha \Delta^{3} u \\
& \quad+\beta \Delta^{2} u-\Delta u=\Delta f(u), \quad x \in \mathbf{R}^{n}, t>0, \\
& u(x, 0)=u_{0}(x), \quad u_{t}(x, 0)=u_{1}(x), \quad x \in \mathbf{R}^{n} .
\end{aligned}
$$

Here $u=u(x, t)$ is the unknown function of $x=\left(x_{1}, \ldots, x_{n}\right) \in \mathbf{R}^{n}$ and $t>0, \gamma, a$, $b, \alpha, \beta$, are positive constants. $f(u)$ is the given nonlinear function, $\Delta^{2} u_{t t}$ and $\Delta u_{t t}$ are the rotational inertia.

The first initial boundary value problem for

$$
u_{t t}-a \Delta u_{t t}-2 b \Delta u_{t}-\alpha \Delta^{3} u+\beta \Delta^{2} u-\Delta u=\gamma \Delta\left(u^{2}\right)
$$

2000 Mathematics Subject Classification. 35L30, 35L75.

Key words and phrases. Generalized damped Boussinesq equation with double rotational inertia, global existence, decay property, time weighted norms technique, pointwise estimates.

* Corresponding author.

Received September 24, 2015. 
in a unit circle was investigated in [28], where $a, b, \alpha, \beta$ are positive constants and $\gamma$ is a constant. The existence and the uniqueness of strong solution was established and the solutions were constructed in the form of series in the small parameter present in the initial conditions. The long-time asymptotic was also obtained in the explicit form. In [7], the authors investigated the IVP for Eq. (1.3) in the unit ball $B \subset \mathbf{R}^{3}$, similar results were established. It is wellknown that the equation Eq. (1.3) is closely contacted with many wave equations. For example, the equation (which we call the $\mathrm{Bq}$ equation)

$$
u_{t t}-u_{x x}+u_{x x x x}=\left(u^{2}\right)_{x x},
$$

which was derived by Boussinesq in 1872 [1] to describe the propagation of long waves with small amplitude on the surface of shallow water. The improved $\mathrm{Bq}$ equation (which we call IBq equation) is

$$
u_{t t}-u_{x x}-u_{x x t t}=\left(u^{2}\right)_{x x} \text {. }
$$

A modification of the IBq equation analogous of the MKdV equation yields

$$
u_{t t}-u_{x x}-u_{x x t t}=\left(u^{3}\right)_{x x}
$$

which we call the IMBq equation (see [8]). In 1-D space, the Cauchy problem for the generalized Boussinesq-type equation has been extensively studied from different views. Lai and $\mathrm{Wu}[6]$ studied the generalized Boussinesq-type equation

$$
u_{t t}-u_{x x}-a u_{x x t t}-2 b u_{t x x}+c u_{x x x}+p u^{2}=\left(u^{3}\right)_{x x}, \quad x \in \mathbf{R}^{1}, t>0 .
$$

They established the global well-posedness in a Sobolov space with a small initial data by means of Fourier transform and the perturbation theory. Wang, $\mathrm{Mu}$ and $\mathrm{Wu}[21]$ employed the scattering theory to study the long-time behavior of small solutions for the generalized Boussinesq-type equation

$$
u_{t t}-u_{x x}+u_{x x x x}-u_{x x t t}+a u_{x x x x t t}=(f(u))_{x x}, \quad x \in \mathbf{R}^{1}, t>0 .
$$

Polat and Ertaş [9], Polat and Pişkin [10] studied the local and global existence, asymptotic behavior and blow up of solutions for

$$
u_{t t}-\Delta u+\Delta^{2} u-\Delta u_{t t}-k \Delta u_{t}=\Delta f(u), \quad x \in \mathbf{R}^{n}, t>0 .
$$

Recently, Shen et al. [12] considered the Cauchy problem for the following 1-D nonlinear wave equation of sixth order

$$
\left\{\begin{array}{l}
u_{t t}+u_{x x x x}+u_{x x x x t t}-\alpha u_{x x}=f\left(u_{x}\right)_{x}, \quad x \in \mathbf{R}, t>0, \\
u(x, 0)=u_{0}(x), \quad u_{t}(x, 0)=u_{1}(x), \quad x \in \mathbf{R} .
\end{array}\right.
$$

They investigated the Cauchy problem of solutions for a class of sixth order 1-D nonlinear wave equations at high initial energy level. By introducing a new stable set They obtained the result that certain solutions with arbitrarily positive initial energy exist globally. Wang and Xue [19] studied the following Cauchy problem for the generalized Boussinesq equation (1-D case):

$$
\left\{\begin{array}{l}
u_{t t}+\alpha u_{x x x x}-u_{x x t t}+u_{x x x x t t}-u_{x x}=f(u)_{x x}, \quad x \in \mathbf{R}, t>0 \\
u(x, 0)=u_{0}(x), \quad u_{t}(x, 0)=u_{1}(x), \quad x \in \mathbf{R}
\end{array}\right.
$$


where $f(u)=u^{p} \quad(p>1)$. They established the existence and uniqueness of the solution and proved the global existence and finite time blowup of the solution to the problem by the potential well method. Quite recently, Chen and Han [4] studied the Cauchy problem for a non-linear wave equation

$$
\begin{cases}u_{t t}-\alpha u_{x x t t}-u_{x x}=g(u)-\alpha g(u)_{x x}, & x \in \mathbf{R}, t>0, \\ u(x, 0)=u_{0}(x), \quad u_{t}(x, 0)=u_{1}(x), & x \in \mathbf{R},\end{cases}
$$

and they proved that the Cauchy problem has a unique global generalized solutions in $C^{2}\left([0, \infty) ; H^{s}(\mathbf{R}) \quad\left(s>\frac{1}{2}\right.\right.$ is a real number $)$ and a unique global classical solution in $C^{2}\left([0, \infty) ; H^{s}(\mathbf{R})\left(s>\frac{5}{2}\right)\right.$. They also proved that the Cauchy problem admits a unique global generalized solution in $C^{2}\left([0, \infty) ; W^{m, p}(\mathbf{R}) \cap\right.$ $L^{\infty}(\mathbf{R})(m \geq 0$ is an integer, $1 \leq p \leq \infty)$ and a unique global classical solution in $C^{2}\left([0, \infty) ; W^{m, p}(\mathbf{R}) \cap L^{\infty}(\mathbf{R})\left(m>2+\frac{1}{p}\right)\right.$.

As for coupled IMBq equations, Wang and $\mathrm{Li}[20]$ considered the Cauchy problem for the following two coupled IMBq equations

$$
\left\{\begin{array}{l}
u_{t t}-\alpha^{2} u_{x x t t}=f(u, v)_{x x}, \quad x \in \mathbf{R}, t>0, \\
v_{t t}-\alpha^{2} v_{x x t t}=g(u, v)_{x x}, \quad x \in \mathbf{R}, t>0, \\
u(x, 0)=u_{0}(x), \quad u_{t}(x, 0)=u_{1}(x), \quad x \in \mathbf{R}, \\
v(x, 0)=v_{0}(x), \quad v_{t}(x, 0)=v_{1}(x), \quad x \in \mathbf{R},
\end{array}\right.
$$

and they proved that, under the assumptions for non-linear terms and initial data, the existence and uniqueness of the global solution and esablished sufficient conditions of blow-up of the solution in finite time by convex methods. This supplements and improves some results by Dé Godefroy [5].

For the space demension $n=1,2,3$ it is worth mentioning that Varlamov [13-17] investigated the local well-posedness and the long-time decay of the Cauchy problem of the generalized Boussinesq-type equation

$$
u_{t t}-2 b \Delta u_{t}+\alpha \Delta^{2} u-\Delta u=\beta \Delta\left(u^{2}\right), \quad x \in \mathbf{R}^{n} \quad(n=1,2,3), t>0 .
$$

We observe that Eq. (1.1) is a higher order wave equation. In higher dimensional space case, Wang and Chen [18] [23] considered the existence, both locally and globally in time, and nonexistence of solutions, and the global existence of small amplitude solutions, and scattering result for the Cauchy problem of the multidimensional generalized IMBq equation

$$
u_{t t}-\Delta u_{t t}-\Delta u=\Delta f(u), \quad x \in \mathbf{R}^{n}, t \geq 0 .
$$

Posteriorly, by employing Besov spaces, some results of [23] were improved by Cho and Ozawa in [2-3]. Recently, Wang and $\mathrm{Xu}$ [24] studied the Cauchy problem for the generalized IBq equation with hydrodynamical damped term

$$
u_{t t}-\Delta u_{t t}-\Delta u-v \Delta u_{t}=\Delta f(u), \quad x \in \mathbf{R}^{n}, t>0 .
$$

They established the optimal decay estimates of solutions under the additional regularity assumption on the initial data. Under smallness condition on the 
initial data, we proved the global existence and decay of the small amplitude solution in the Sobolev space. Later on, Wang and Da [25] improved some results of [2-3] [18] [23-24]. More precisely, they investigated the IVP associated with the generalized damped Boussinesq equation with double dispersion

$$
u_{t t}-\Delta u_{t t}-b \Delta u_{t}+\Delta^{2} u-a \Delta u=\Delta f(u), \quad x \in \mathbf{R}^{n}, t>0 .
$$

Based on decay estimates of solutions to the corresponding linear equation, they established the decay estimates and the pointwise estimates by using Fourier transform and multiplier method. Under small condition on the initial data, they obtained the existence and asymptotic behavior of global solutions in the corresponding Sobolev spaces by time weighted norms technique and the contraction mapping principle. Recently, in the case of $\gamma=1, a=1, b=0, \alpha=0$ and $\beta=1$ in (1.1), Xia and Yuan [26] considered the following Cauchy problem of a Boussinesq type equation of sixth order

$$
u_{t t}+\Delta^{2} u_{t t}-\Delta u_{t t}+\beta \Delta^{2} u-\Delta u=\Delta f(u), \quad x \in \mathbf{R}^{n}, t>0 .
$$

They investigated the existence and uniqueness of the global small solution to (1.4) and (1.2) as well as the small data scattering result to the Cauchy problem for a Boussinesq type equation of (1.4) and (1.2) with the nonlinear term $f(u)$ behaving as $u^{p}(p>1)$ as $u \rightarrow 0$ in $\mathbf{R}^{n}(n \geq 1)$. The main method and techniques used in their paper are the Littlewood-Paley dyadic decomposition, the stationary phase estimate and some properties of Bessel function. Quite recently, Pişkin and Polat [11] considered the Cauchy problem of the generalized multidimensional Boussinesq-type equation with a damping term

$$
\begin{aligned}
& u_{t t}+\Delta^{2} u_{t t}-\Delta u_{t t}-k \Delta u_{t}+\Delta^{2} u-\Delta u=\Delta f(u), \quad x \in \mathbf{R}^{n}, t>0, \\
& u(x, 0)=u_{0}(x), \quad u_{t}(x, 0)=u_{1}(x), \quad x \in \mathbf{R}^{n},
\end{aligned}
$$

and obtained the existence, both locally and globally in time, the global nonexistence, and the asymptotic behavior of solutions for (1.5).

The main purpose of this paper is to establish global existence and asymptotic behavior of solutions to (1.1) and (1.2) by using the contraction mapping principle. Firstly, we consider the decay property of the following linear equation

$$
u_{t t}-\gamma \Delta^{2} u_{t t}-a \Delta u_{t t}-2 b \Delta u_{t}-\alpha \Delta^{3} u+\beta \Delta^{2} u-\Delta u=0 .
$$

Then we obtain the following decay estimate of solutions to (1.5) associated with initial condition (1.2),

$$
\begin{aligned}
& \left\|\partial_{x}^{k} u(t)\right\|_{L^{2}} \leq C(1+t)^{-n / 4-k / 2-1 / 2}\left(\left\|u_{0}\right\|_{\dot{H}_{1}^{-1}}+\left\|u_{1}\right\|_{\dot{H}_{1}^{-2}}+\left\|u_{0}\right\|_{H^{s+2}}+\left\|u_{1}\right\|_{H^{s}}\right) \\
& \quad(k \leq s+2) \\
& \left\|\partial_{x}^{h} u_{t}(t)\right\|_{L^{2}} \leq C(1+t)^{-n / 4-h / 2-1}\left(\left\|u_{0}\right\|_{\dot{H}_{1}^{-1}}+\left\|u_{1}\right\|_{\dot{H}_{1}^{-2}}+\left\|u_{0}\right\|_{H^{s+2}}+\left\|u_{1}\right\|_{H^{s}}\right) \\
& \quad(h \leq s)
\end{aligned}
$$


Based on the estimates (1.7) and (1.8), we establish global existence and decay estimates of solutions to (1.1) and (1.2) by using time weighted norm technique and the contraction mapping principle. This result improves earlier ones in the literature, such as [9] [19] [25].

To facilitate further on our analysis, we present some notations which will be used throughout in our contribution. Let $\mathscr{F}[u]$ denote the Fourier transform of $u$ defined by

$$
\hat{u}(\xi)=\mathscr{F}[u]=\int_{\mathbf{R}^{n}} e^{-i \xi \cdot x} u(x) d x,
$$

and we denote its inverse transform by $\mathscr{F}^{-1}$.

For $1 \leq p \leq \infty, L^{p}=L^{p}\left(\mathbf{R}^{n}\right)$ denotes the usual Lebesgue space with the norm $\|\cdot\|_{L^{p}}$. Recall that $J_{s}=(I-\Delta)^{s / 2}$ and $I_{s}=(-\Delta)^{s / 2}$ are said to be the Bessel and the Riesz potentials, respectively. Assume that $1<p<\infty,-\infty<$ $s<\infty$. The usual Sobolev space of $s$ is defined by $H_{p}^{s}=(I-\Delta)^{-s / 2} L^{p}$ (Bessel potential spaces) with the norm $\|f\|_{H_{s}^{s}}=\left\|(I-\Delta)^{s / 2} f\right\|_{L^{p}}$; the homogeneous Sobolev space of $s$ is defined by $\dot{H}_{p}^{s}=(-\Delta)^{-s / 2} L^{p}$ (Riesz potential spaces) with the norm $\|f\|_{H_{p}^{s}}=\left\|(-\Delta)^{s / 2} f\right\|_{L^{p}}$; especially $H^{s}=H_{2}^{s}, \dot{H}^{s}=\dot{H}_{2}^{s}$. Moreover, we know that $H_{p}^{s}=L^{p} \cap \dot{H}_{p}^{s}$ for $s \geq 0$. More details are present in the Wang's et al. book [22].

We use $A \sim B$ using the statement that $A \leq C_{1} B$ and $B \leq C_{1} A$ for some constant $C_{1}>0$, use $A \ll B$ to denote the statement that $A \leq \frac{1}{C_{2}} B$ for some large enough constant $C_{2}>0$. We denote every positive constant by the same symbol $C$ or $c$ without confusion. [.] is the Gauss symbol.

The rest of the paper is organized as follows. In section 2, we establish the pointwise estimates of the solutions. Then, in Section 3, we discuss the linear problem and show the decay estimates. Finally, we prove global existence and asymptotic behavior of solutions for the Cauchy problem (1.1) and (1.2) in Section 5 .

\section{Pointwise estimates of the solutions}

The aim of this section is to establish the pointwise estimates of the solutions. Before proceeding to our analysis, we obtain the solution formula for the Cauchy problem (1.1) and (1.2). we have

We first investigate the linear equation (1.4). Taking the Fourier transform,

$$
\left(1+a|\xi|^{2}+\gamma|\xi|^{4}\right) \hat{u}_{t t}+2 b|\xi|^{2} \hat{u}_{t}+\left(\alpha|\xi|^{6}+\beta|\xi|^{4}+|\xi|^{2}\right) \hat{u}=0 .
$$

The corresponding initial value are

$$
t=0: \quad \hat{u}=\hat{u}_{0}(\xi), \quad \hat{u}_{t}=\hat{u}_{1}(\xi) .
$$


The characteristic equation of (2.1) is

$$
\left(1+a|\xi|^{2}+\gamma|\xi|^{4}\right) \lambda^{2}+2 b|\xi|^{2} \lambda+\alpha|\xi|^{6}+\beta|\xi|^{4}+|\xi|^{2}=0
$$

Let $\lambda=\lambda_{ \pm}(\xi)$ be the corresponding eigenvalues of (2.3), we obtain

$$
=\frac{-b|\xi|^{2} \pm|\xi| \sqrt{-1-\left(a+\beta-b^{2}\right)|\xi|^{2}-(\alpha+a \beta+\gamma)|\xi|^{4}-(a \alpha+\beta \gamma)|\xi|^{6}-\alpha \gamma|\xi|^{8}}}{1+a|\xi|^{2}+\gamma|\xi|^{4}} .
$$

The solution to the problem (2.1) and (2.2) is given in the form

$$
\hat{u}(\xi, t)=\hat{G}(\xi, t) \hat{u}_{1}(\xi)+\hat{H}(\xi, t) \hat{u}_{0}(\xi)
$$

where

$$
\hat{G}(\xi, t)=\frac{1}{\lambda_{+}(\xi)-\lambda_{-}(\xi)}\left(e^{\lambda_{+}(\xi) t}-e^{\lambda_{-}(\xi) t}\right)
$$

and

$$
\hat{H}(\xi, t)=\frac{1}{\lambda_{+}(\xi)-\lambda_{-}(\xi)}\left(\lambda_{+}(\xi) e^{\lambda_{-}(\xi) t}-\lambda_{-}(\xi) e^{\lambda_{+}(\xi) t}\right) .
$$

By the Duhamel principle and (2.5), we obtain the solution formula to (2.1) and (2.2),

$$
u(t)=G(t) * u_{1}+H(t) * u_{0}+\int_{0}^{t} G(t-\tau) *\left(I-a \Delta+\gamma \Delta^{2}\right)^{-1} \Delta f(u)(\tau) d \tau .
$$

where

$$
G(x, t)=\mathscr{F}^{-1}[\hat{G}(\xi, t)](x), \quad H(x, t)=\mathscr{F}^{-1}[\hat{H}(\xi, t)](x) .
$$

Next, we shall establish the pointwise estimates of the solutions.

LEMMA 2.1. The solution of problem (2.1) and (2.2) satisfies

$$
\begin{aligned}
& |\xi|^{2}\left(1+|\xi|^{2}+|\xi|^{4}\right)|\hat{u}(\xi, t)|^{2}+\left|\hat{u}_{t}(\xi, t)\right|^{2} \\
& \quad \leq C e^{-c \rho(\xi) t}\left[|\xi|^{2}\left(1+|\xi|^{2}+|\xi|^{4}\right)\left|\hat{u}_{0}(\xi)\right|^{2}+\left|\hat{u}_{1}(\xi)\right|^{2}\right],
\end{aligned}
$$

for $\xi \in \mathbf{R}^{n}$ and $t>0$, where $\rho(\xi)=\frac{|\xi|^{2}}{1+|\xi|^{2}+|\xi|^{4}}$.

Proof. Multiplying (2.1) by $\overline{\hat{u}}_{t}$ and taking the real part yields (2.10) $\frac{1}{2} \frac{d}{d t}\left\{\left(1+a|\xi|^{2}+\gamma|\xi|^{4}\right)\left|\hat{u}_{t}\right|^{2}+\left(\alpha|\xi|^{6}+\beta|\xi|^{4}+|\xi|^{2}\right)|\hat{u}|^{2}\right\}+2 b|\xi|^{2}\left|\hat{u}_{t}\right|^{2}=0$. 
Multiplying (2.1) by $\overline{\hat{u}}$ and taking the real part, we obtain

$$
\begin{aligned}
& \frac{1}{2} \frac{d}{d t}\left\{b|\xi|^{2}|\hat{u}|^{2}+2\left(1+a|\xi|^{2}+\gamma|\xi|^{4}\right) \operatorname{Re}\left(\hat{u}_{t} \overline{\hat{u}}\right)\right\} \\
& \quad+\left(\alpha|\xi|^{6}+\beta|\xi|^{4}+|\xi|^{2}\right)|\hat{u}|^{2}-\left(1+a|\xi|^{2}+\gamma|\xi|^{4}\right)\left|\hat{u}_{t}\right|^{2}=0 .
\end{aligned}
$$

Multiplying both sides of $(2.10)$ and $(2.11)$ by $\left(1+a|\xi|^{2}+\gamma|\xi|^{4}\right)$ and $b|\xi|^{2}$ respectively, summing up the products yields

$$
\frac{d}{d t} E+F=0
$$

where

$$
\begin{aligned}
E= & \frac{1}{2}\left(1+a|\xi|^{2}+\gamma|\xi|^{4}\right)\left|\hat{u}_{t}\right|^{2}+\left(1+a|\xi|^{2}+\gamma|\xi|^{4}\right)\left(\alpha|\xi|^{6}+\beta|\xi|^{4}+|\xi|^{2}\right)|\hat{u}|^{2} \\
& +b^{2}|\xi|^{4}|\hat{u}|^{2}+b|\xi|^{2}\left(1+a|\xi|^{2}+\gamma|\xi|^{4}\right) \operatorname{Re}\left(\hat{u}_{t} \overline{\hat{u}}\right)
\end{aligned}
$$

and

$$
F=b|\xi|^{2}\left(\alpha|\xi|^{6}+\beta|\xi|^{4}+|\xi|^{2}\right)|\hat{u}|^{2}+b|\xi|^{2}\left(1+a|\xi|^{2}+\gamma|\xi|^{4}\right)\left|\hat{u}_{t}\right|^{2} .
$$

A simple computation implies that

$$
E \sim\left(1+a|\xi|^{2}+\gamma|\xi|^{4}\right) E_{0}
$$

where

$$
E_{0}=|\xi|^{2}\left(1+|\xi|^{2}+|\xi|^{4}\right)|\hat{u}|^{2}+\left|\hat{u}_{t}\right|^{2} .
$$

Note that $F \gtrsim c|\xi|^{2} E_{0}$. It follows from (2.13) that

$$
F \gtrsim \rho(\xi) E,
$$

where

$$
\rho(\xi)=\frac{|\xi|^{2}}{1+|\xi|^{2}+|\xi|^{4}} .
$$

By (2.12) and (2.14), we get

$$
\frac{d}{d t} E+c \rho(\xi) E \leq 0
$$

Thus $E(\xi, t) \leq e^{-c \rho(\xi) t} E(\xi, 0)$, which together with (2.13) proves the desired estimates (2.9). Then the proof is complete.

Lemma 2.2. Let $\hat{\boldsymbol{G}}(\xi, t)$ and $\hat{H}(\xi, t)$ be the fundamental solution of (2.5) in the Fourier space, which are given in (2.6) and (2.7), respectively. Then we have the estimates

$$
|\xi|^{2}\left(1+|\xi|^{2}+|\xi|^{4}\right)|\hat{\boldsymbol{G}}(\xi, t)|^{2}+\left|\hat{\boldsymbol{G}}_{t}(\xi, t)\right|^{2} \leq C e^{-c \rho(\xi) t}
$$


and

$$
|\xi|^{2}\left(1+|\xi|^{2}+|\xi|^{4}\right)|\hat{H}(\xi, t)|^{2}+\left|\hat{H}_{t}(\xi, t)\right|^{2} \leq C|\xi|^{2}\left(1+|\xi|^{2}+|\xi|^{4}\right) e^{-c \rho(\xi) t}
$$

for $\xi \in \mathbf{R}^{n}$ and $t \geq 0$, where $\rho(\xi)=\frac{|\xi|^{2}}{1+|\xi|^{2}+|\xi|^{4}}$.

Proof. If $\hat{u}_{0}(\xi)=0$, from (2.5), we have

$$
\hat{u}(\xi, t)=\hat{G}(\xi, t) \hat{u}_{1}(\xi), \quad \hat{u}_{t}(\xi, t)=\hat{G}_{t}(\xi, t) \hat{u}_{1}(\xi) .
$$

Substituting the equalities into (2.9) with $\hat{u}_{0}(\xi)=0$, we obtain (2.15). In what follows, we consider $\hat{u}_{1}(\xi)=0$, it follows from (2.5) that

$$
\hat{u}(\xi, t)=\hat{H}(\xi, t) \hat{u}_{0}(\xi), \quad \hat{u}_{t}(\xi, t)=\hat{H}_{t}(\xi, t) \hat{u}_{0}(\xi) .
$$

Substituting the equalities into $(2.9)$ with $\hat{u}_{1}(\xi)=0$, we obtain the desired estimate (2.16). The Lemma is proved.

Lemma 2.3. Let $k \geq 0$ and $1 \leq p \leq 2$. Then we have

$$
\begin{aligned}
\left\|\partial_{x}^{k} G(t) * \phi\right\|_{L^{2}} \leq & C(1+t)^{-(n / 2(1 / p-1 / 2)+k / 2+l / 2-1 / 2)}\|\phi\|_{\dot{H}_{p}^{-l}} \\
& +C e^{-c t}\left\|\partial_{x}^{(k-2)+} \phi\right\|_{L^{2}}, \\
\left\|\partial_{x}^{k} H(t) * \phi\right\|_{L^{2}} \leq & C(1+t)^{-(n / 2(1 / p-1 / 2)+k / 2+l / 2)}\|\phi\|_{\dot{H}_{p}^{-l}} \\
& +C e^{-c t}\left\|\partial_{x}^{k} \phi\right\|_{L^{2}} \\
\left\|\partial_{x}^{k} G_{t}(t) * \phi\right\|_{L^{2}} \leq & C(1+t)^{-(n / 2(1 / p-1 / 2)+k / 2+l / 2)}\|\phi\|_{\dot{H}_{p}^{-l}} \\
& +C e^{-c t}\left\|\partial_{x}^{k} \phi\right\|_{L^{2}} \\
\left\|\partial_{x}^{k} H_{t}(t) * \phi\right\|_{L^{2}} \leq & C(1+t)^{-(n / 2(1 / p-1 / 2)+k / 2+l / 2+1 / 2)}\|\phi\|_{\dot{H}_{p}^{-l}} \\
& +C e^{-c t}\left\|\partial_{x}^{k+2} \phi\right\|_{L^{2}} \\
\| \partial_{x}^{k} G(t) *(I-a \Delta+ & \left.\gamma \Delta^{2}\right)^{-1} \Delta g\left\|_{L^{2}} \leq C(1+t)^{-(n / 4+k / 2+1 / 2)}\right\| g \|_{L^{1}} \\
& +C e^{-c t}\left\|\partial_{x}^{k} g\right\|_{L^{2}}, \\
\left\|\partial_{x}^{k} G_{t}(t) *(I-a \Delta)^{-1} \Delta g\right\|_{L^{2}} \leq C(1+t)^{-(n / 4+k / 2+1)}\|g\|_{L^{1}} & +C e^{-c t}\left\|\partial_{x}^{k} g\right\|_{L^{2}}
\end{aligned}
$$

where $(k-2)_{+}=\max \{0, k-2\}$.

Proof. Firstly, we prove (2.17). We divide the integral into two parts corresponding to the low frequency region $|\xi| \lesssim 1$ and the high frequency region $|\xi| \gtrsim 1$. Using Plancherel's identity [22] and (2.15), we obtain 


$$
\begin{aligned}
\left\|\partial_{x}^{k} G(t) * \phi\right\|_{L^{2}}^{2} & \int_{|\xi| \lesssim 1}|\xi|^{2 k}|\hat{G}(\xi, t)|^{2}|\hat{\phi}(\xi)|^{2} d \xi+\int_{|\xi| \gtrsim 1}|\xi|^{2 k}|\hat{G}(\xi, t)|^{2}|\hat{\phi}(\xi)|^{2} d \xi \\
\leq & C \int_{|\xi| \lesssim 1}|\xi|^{2 k-2} e^{-c|\xi|^{2} t}|\hat{\phi}(\xi)|^{2} d \xi \\
& +C e^{-c t} \int_{|\xi| \gtrsim 1}|\xi|^{2 k}\left(|\xi|^{2}\left(1+|\xi|^{2}+|\xi|^{4}\right)\right)^{-1}|\hat{\phi}(\xi)|^{2} d \xi \\
\leq & C\left\||\xi|^{-l} \hat{\phi}(\xi)\right\|_{L^{p^{\prime}}}^{2}\left(\int_{|\xi| \lesssim 1}|\xi|^{(2 k-2+2 l) q} e^{-c q|\xi|^{2} t} d \xi\right)^{1 / q} \\
& +C e^{-c t}\left\|\partial_{x}^{(k-2)_{+}} \phi\right\|_{L^{2}}^{2},
\end{aligned}
$$

where $\frac{1}{p}+\frac{1}{p^{\prime}}=1, \frac{2}{p^{\prime}}+\frac{1}{q}=1$. It follows from Hausdorff-Young inequality [2]

$$
\left\||\xi|^{-l} \hat{\phi}(\xi)\right\|_{L^{p^{\prime}}} \leq C\left\|(-\Delta)^{-l / 2} \phi\right\|_{L^{p}} .
$$

By the inequality

$$
\begin{gathered}
\int_{|\xi| \leq \delta}|\xi|^{k} e^{-c|\xi|^{2} t} d \xi \lesssim(1+t)^{-(k+n) / 2}, \quad \forall t \geq 0, \\
\sup _{0 \leq|\xi| \leq \delta}|\xi|^{k} e^{-c|\xi|^{2} t} d \xi \lesssim(1+t)^{-k / 2}, \quad \forall t \geq 0,
\end{gathered}
$$

in $[18$, pp. 1184], we obtain

$$
\begin{aligned}
\left(\int_{|\xi| \lesssim 1}|\xi|^{(2 k-2+2 l) q} e^{-c q|\xi|^{2} t} d \xi\right)^{1 / q} & \leq C(1+t)^{-(n / 2 q+k-1+l)} \\
& \leq C(1+t)^{-(n(1 / p-1 / 2)+k-1+l)} .
\end{aligned}
$$

Combining (2.23), (2.24) and (2.25) yields (2.17).

Similarly, using (2.15) and (2.16), respectively, we can prove (2.18)-(2.20).

Next, we prove (2.21). By the Plancherel theorem, (2.15) and HausdorffYoung inequality, we have

$$
\begin{aligned}
& \left\|\partial_{x}^{k} G(t) *\left(I-a \Delta+\gamma \Delta^{2}\right)^{-1} \Delta g\right\|_{L^{2}}^{2} \\
& =\int_{|\xi| \lesssim 1}|\xi|^{2 k}|\hat{G}(\xi, t)|^{2}|\xi|^{4}\left(1+|\xi|^{2}+|\xi|^{4}\right)^{-2}|\hat{g}(\xi)|^{2} d \xi \\
& \quad+\int_{|\xi| \gtrsim 1}|\xi|^{2 k}|\hat{G}(\xi, t)|^{2}|\xi|^{4}\left(1+|\xi|^{2}+|\xi|^{4}\right)^{-2}|\hat{g}(\xi)|^{2} d \xi
\end{aligned}
$$




$$
\begin{aligned}
& \leq C \int_{|\xi| \lesssim 1}|\xi|^{2 k+2} e^{-c|\xi|^{2} t}|\hat{g}(\xi)|^{2} d \xi+C e^{-c t} \int_{|\xi| \gtrsim 1}|\xi|^{2 k}|\hat{g}(\xi)|^{2} d \xi \\
& \leq C\|\hat{g}(\xi)\|_{L^{\infty}}^{2} \int_{|\xi| \lesssim 1}|\xi|^{2 k+2} e^{-c|\xi|^{2} t} d \xi+C e^{-c t}\left\|\partial_{x}^{k} g\right\|_{L^{2}}^{2} \\
& \leq C(1+t)^{-(n / 2+k+1)}\|g\|_{L^{1}}^{2}+C e^{-c t}\left\|\partial_{x}^{k} g\right\|_{L^{2}}^{2} .
\end{aligned}
$$

Thus (2.21) follows. Similarly, we can prove (2.22). Thus we have completed the proof of Lemma 2.3.

\section{Decay estimates for solutions to the linear equation}

THEOREM 3.1. Assume that $u_{0} \in H^{s+2}\left(\mathbf{R}^{n}\right) \cap \dot{H}_{1}^{-1}\left(\mathbf{R}^{n}\right), \quad u_{1} \in H^{s}\left(\mathbf{R}^{n}\right) \cap$ $\dot{H}_{1}^{-2}\left(\mathbf{R}^{n}\right)\left(s \geq\left[\frac{n}{2}\right]+5\right)$. Then the classical solution $u(x, t)$ to (1.4) associated with initial data (1.2), satisfies the decay estimates

$$
\left\|\partial_{x}^{k} u(t)\right\|_{L^{2}} \lesssim(1+t)^{-n / 4-k / 2-1 / 2}\left(\left\|u_{0}\right\|_{\dot{H}_{1}^{-1}}+\left\|u_{1}\right\|_{\dot{H}_{1}^{-2}}+\left\|u_{0}\right\|_{H^{s+2}}+\left\|u_{1}\right\|_{H^{s}}\right)
$$

for $k \leq s+2$,

$$
\left\|\partial_{x}^{h} u_{t}(t)\right\|_{L^{2}} \lesssim(1+t)^{-n / 4-h / 2-1}\left(\left\|u_{0}\right\|_{\dot{H}_{1}^{-1}}+\left\|u_{1}\right\|_{\dot{H}_{1}^{-2}}+\left\|u_{0}\right\|_{H^{s+2}}+\left\|u_{1}\right\|_{H^{s}}\right)
$$

for $h \leq s$,

$$
\left\|\partial_{x}^{m} u(t)\right\|_{L^{\infty}} \lesssim(1+t)^{-n / 2-m / 2-1 / 2}\left(\left\|u_{0}\right\|_{\dot{H}_{1}^{-1}}+\left\|u_{1}\right\|_{\dot{H}_{1}^{-2}}+\left\|u_{0}\right\|_{H^{s+2}}+\left\|u_{1}\right\|_{H^{s}}\right)
$$

for $m \leq s+1-\left[\frac{n}{2}\right]$.

Proof. Firstly, we prove (3.1). It follows from (2.17) and (2.18) that

$$
\begin{aligned}
\left\|\partial_{x}^{k} u(t)\right\|_{L^{2}} & \lesssim\left\|\partial_{x}^{k} G(t) * u_{1}\right\|_{L^{2}}+\left\|\partial_{x}^{h} H(t) * u_{0}\right\|_{L^{2}} \\
& \lesssim(1+t)^{-n / 4-k / 2-1 / 2}\left(\left\|u_{0}\right\|_{\dot{H}_{1}^{-1}}+\left\|u_{1}\right\|_{\dot{H}_{1}^{-2}}\right)+e^{-c t}\left(\left\|u_{0}\right\|_{H^{s+2}}+\left\|u_{1}\right\|_{H^{s}}\right) \\
& \lesssim(1+t)^{-n / 4-k / 2-1 / 2}\left(\left\|u_{0}\right\|_{\dot{H}_{1}^{-1}}+\left\|u_{1}\right\|_{\dot{H}_{1}^{-2}}+\left\|u_{0}\right\|_{H^{s+2}}+\left\|u_{1}\right\|_{H^{s}}\right) .
\end{aligned}
$$

Similar to the proof of (3.1), using (2.19) and (2.20), we obtain (3.2). Next, we prove (3.3). Using (3.1) and Gagliardo-Nirenberg inequality [22], it is not difficult to get (3.3). Hence, we omit the procedure here. The Lemma is complete.

\section{Existence of global solution and asymptotic behavior}

In this section, we prove the existence and asymptotic behavior of global solutions to the Cauchy problem (1.1) and (1.2). Before going to our main result, we need the following technical Lemma. 
Lemma 4.1 [27]. Let $s$ and $\theta$ be positive integers, $\delta>0, p, q, r \in[1, \infty]$ satisfy $\frac{1}{r}=\frac{1}{p}+\frac{1}{r}$, and let $k \in\{0,1,2, \ldots, s\}$. Assume that $F(v)$ is a class of $C^{s}$ and satisfies

$$
\left|\partial_{v}^{l} F(v)\right| \leq C_{l, \delta}|v|^{\theta+1-l}, \quad|v| \leq \delta, 0 \leq l \leq s, l<\theta+1
$$

and

$$
\left|\partial_{v}^{l} F(v)\right| \leq C_{l, \delta}, \quad|v| \leq \delta, l \leq s, \theta+1 \leq l .
$$

If $v \in L^{p} \cap W^{k, q} \cap L^{\infty}$ and $\|v\|_{L^{\infty}} \leq \delta$, then

$$
\begin{gathered}
\|F(v)\|_{W^{k, r}} \leq C_{k, \delta}\|v\|_{W^{k, q}}\|v\|_{L^{p}}\|v\|_{L^{\infty}}^{\theta-1}, \\
\left\|\partial_{x}^{\alpha} F(v)\right\|_{L^{r}} \leq C_{k, \delta}\left\|\partial_{x}^{\alpha} v\right\|_{L^{q}}\|v\|_{L^{p}}\|v\|_{L^{\infty}}^{\theta-1}, \quad|\alpha| \leq k .
\end{gathered}
$$

Lemma 4.2 [27]. Let $s$ and $\theta$ be positive integers, $\delta>0, p, q, r \in[1, \infty]$ satisfy $\frac{1}{r}=\frac{1}{p}+\frac{1}{r}$, and let $k \in\{0,1,2, \ldots, s\}$. Let $F(v)$ be a function that satisfies the assumptions of Lemma 4.1. Moreover, assume that

$$
\left|\partial_{v}^{s} F\left(v_{1}\right)-\partial_{v}^{s} F\left(v_{2}\right)\right| \leq C_{\delta}\left(\left|v_{1}\right|+\left|v_{2}\right|\right)^{\max \{\theta-s, \theta\}}\left|v_{1}-v_{2}\right|, \quad\left|v_{1}\right| \leq \delta, \quad\left|v_{2}\right| \leq \delta .
$$

If $v_{1}, v_{2} \in L^{p} \cap W^{k, q} \cap L^{\infty}$ and $\left\|v_{1}\right\|_{L^{\infty}} \leq \delta,\left\|v_{2}\right\|_{L^{\infty}} \leq \delta$, then for $|\alpha| \leq k$, we have

$$
\begin{aligned}
\left\|\partial_{x}^{\alpha}\left(F\left(v_{1}\right)-F\left(v_{2}\right)\right)\right\|_{L^{r}} & \\
\leq & C_{k, \delta}\left\{\left(\left\|\partial_{x}^{\alpha} v_{1}\right\|_{L^{q}}+\left\|\partial_{x}^{\alpha} v_{2}\right\|_{L^{q}}\right)\left\|v_{1}-v_{2}\right\|_{L^{p}}\right. \\
& \left.\quad+\left(\left\|v_{1}\right\|_{L^{p}}+\left\|v_{2}\right\|_{L^{p}}\right)\left\|\partial_{x}^{\alpha}\left(v_{1}-v_{2}\right)\right\|_{L^{q}}\right\}\left(\left\|v_{1}\right\|_{L^{\infty}}+\left\|v_{2}\right\|_{L^{\infty}}\right)^{\theta-1} .
\end{aligned}
$$

Based on the estimates (3.1)-(3.3) of solutions to (1.4) with respect to initial data (1.2), we define the following Banach space

$$
X=\left\{u \in C\left([0, \infty) ; H^{s+2}\left(\mathbf{R}^{n}\right)\right) \cap C^{1}\left([0, \infty) ; H^{s}\left(\mathbf{R}^{n}\right)\right):\|u\|_{X}<\infty\right\},
$$

where the time weighted norm

$$
\|u\|_{X}=\sup _{t \geq 0}\left\{\sum_{k \leq s+2}(1+t)^{n / 4+k / 2+1 / 2}\left\|\partial_{x}^{k} u(t)\right\|_{L^{2}}+\sum_{h \leq s}(1+t)^{n / 4+h / 2+1}\left\|\partial_{x}^{h} u_{t}(t)\right\|_{L^{2}}\right\}
$$

For $R>0$, we define $X_{R}=\left\{u \in X:\|u\|_{X} \leq R\right\}$. For $m \leq s+1-\left[\frac{n}{2}\right]$, using
Gagliardo-Nirenberg inequality, we get

$$
\left\|\partial_{x}^{m} u(t)\right\|_{L^{\infty}} \leq C(1+t)^{-(n / 2+m / 2+1 / 2)}\|u\|_{X} .
$$

We now are position to state the main results. 
THEOREM 4.3. Assume that $u_{0} \in H^{s+2}\left(\mathbf{R}^{n}\right) \cap \dot{H}_{1}^{-1}\left(\mathbf{R}^{n}\right), \quad u_{1} \in H^{s}\left(\mathbf{R}^{n}\right) \cap$ $\dot{H}_{1}^{-2}\left(\mathbf{R}^{n}\right)\left(s \geq\left[\frac{n}{2}\right]+5\right)$ and integer $\theta \geq 2$. Let $f(u)$ be a function of class $C^{s+2}$ and satisfy Lemmas 4.1 and 4.2. Let

$$
E_{0}=\left\|u_{0}\right\|_{\dot{H}_{1}^{-1}}+\left\|u_{1}\right\|_{\dot{H}_{1}^{-2}}+\left\|u_{0}\right\|_{H^{s+2}}+\left\|u_{1}\right\|_{H^{s}} .
$$

If $E_{0}$ is suitably small, the Cauchy problem (1.1) and (1.2) has a unique global classical solution $u(x, t)$ satisfying $u \in C\left([0, \infty) ; H^{s+2}\left(\mathbf{R}^{n}\right)\right), u_{t} \in C\left([0, \infty) ; H^{s}\left(\mathbf{R}^{n}\right)\right)$, $u_{t t} \in L^{\infty}\left([0, \infty) ; H^{s-2}\left(\mathbf{R}^{n}\right)\right)$. Moreover, the solution satisfies the decay estimate

$$
\begin{gathered}
\left\|\partial_{x}^{k} u(t)\right\|_{L^{2}} \lesssim E_{0}(1+t)^{-n / 4-k / 2-1 / 2} \\
\left\|\partial_{x}^{h} u_{t}(t)\right\|_{L^{2}} \lesssim E_{0}(1+t)^{-n / 4-h / 2-1}
\end{gathered}
$$

for $k \leq s+2$ and $h \leq s$.

Proof. Let us define the nonlinear mapping (4.4) $\Phi(u)=G(t) * u_{1}+H(t) * u_{0}+\int_{0}^{t} G(t-\tau) *\left(I-a \Delta+\gamma \Delta^{2}\right)^{-1} \Delta f(u(\tau)) d \tau$.

From (2.17), (2.18), (2.21), Lemma 4.1 and (4.1), for $k \leq s+2$, we conclude

$$
\begin{aligned}
&\left\|\partial_{x}^{k} \Phi(u)\right\|_{L^{2}} \\
& \leq C\left\|\partial_{x}^{k} G(t) * u_{1}\right\|_{L^{2}}+C\left\|\partial_{x}^{k} H(t) * u_{0}\right\|_{L^{2}} \\
&+C \int_{0}^{t}\left\|\partial_{x}^{k} G(t-\tau) *\left(I-a \Delta+\gamma \Delta^{2}\right)^{-1} \Delta f(u(\tau))\right\|_{L^{2}} d \tau \\
& \leq C(1+t)^{-n / 4-k / 2-1 / 2}\left(\left\|u_{0}\right\|_{\dot{H}_{1}^{-1}}+\left\|u_{1}\right\|_{\dot{H}_{1}^{-2}}\right)+C e^{-c t}\left(\left\|u_{0}\right\|_{H^{s+2}}+\left\|u_{1}\right\|_{H^{s}}\right) \\
&+C \int_{0}^{t / 2}(1+t-\tau)^{-n / 4-k / 2-1 / 2}\|f(u)\|_{L^{1}} d \tau \\
&+C \int_{t / 2}^{t}(1+t-\tau)^{-n / 4-1 / 2}\left\|\partial_{x}^{k} f(u)\right\|_{L^{1}} d \tau \\
&+C \int_{0}^{t} e^{-c(t-\tau)}\left\|\partial_{x}^{k} f(u)\right\|_{L^{2}} d \tau \\
& \leq C(1+t)^{-n / 4-k / 2-1 / 2}\left(\left\|u_{0}\right\|_{\dot{H}_{1}^{-1}}+\left\|u_{1}\right\|_{\dot{H}_{1}^{-2}}\right)+C e^{-c t}\left(\left\|u_{0}\right\|_{H^{s+2}}+\left\|u_{1}\right\|_{H^{s}}\right) \\
&+C \int_{0}^{t / 2}(1+t-\tau)^{-n / 4-k / 2-1 / 2}\|u\|_{L^{2}}^{2}\|u\|_{L^{\infty}}^{\theta-1} d \tau \\
&+C \int_{t / 2}^{t}(1+t-\tau)^{-n / 4-1 / 2}\left\|\partial_{x}^{k} u\right\|_{L^{2}}^{2}\|u\|_{L^{\infty}}^{\theta-1} d \tau+C \int_{0}^{t} e^{-c(t-\tau)}\left\|\partial_{x}^{k} u\right\|_{L^{2}}\|u\|_{L^{\infty}}^{\theta} d \tau
\end{aligned}
$$




$$
\begin{aligned}
\leq & C(1+t)^{-n / 4-k / 2-1 / 2}\left(\left\|u_{0}\right\|_{\dot{H}_{1}^{-1}}+\left\|u_{1}\right\|_{\dot{H}_{1}^{-2}}\right)+C e^{-c t}\left(\left\|u_{0}\right\|_{H^{s+2}}+\left\|u_{1}\right\|_{H^{s}}\right) \\
& +C R^{\theta+1} \int_{0}^{t / 2}(1+t-\tau)^{-n / 4-k / 2-1 / 2}(1+\tau)^{-(n / 2+1)}(1+\tau)^{-(n / 2+1 / 2)(\theta-1)} d \tau \\
& +C R^{\theta+1} \int_{t / 2}^{t}(1+t-\tau)^{-n / 4-1 / 2}(1+\tau)^{-n / 2-k-1}(1+\tau)^{-(n / 2+1 / 2)(\theta-1)} d \tau \\
& +C R^{\theta+1} \int_{0}^{t} e^{-c(t-\tau)}(1+\tau)^{-n / 4-k / 2-1 / 2}(1+\tau)^{-(n / 2+1 / 2) \theta} d \tau \\
\leq & C(1+t)^{-n / 4-k / 2-1 / 2}\left\{\left(\left\|u_{0}\right\|_{\dot{H}_{1}^{-1}}+\left\|u_{1}\right\|_{\dot{H}_{1}^{-2}}+\left\|u_{0}\right\|_{H^{s+2}}+\left\|u_{1}\right\|_{H^{s}}\right)+R^{\theta+1}\right\} .
\end{aligned}
$$

Thus

$$
(1+t)^{n / 4+k / 2+1 / 2}\left\|\partial_{x}^{k} \Phi(u)\right\|_{L^{2}} \leq C E_{0}+C R^{\theta+1} .
$$

It follows from (4.4) that

$$
\begin{aligned}
\Phi(u)_{t}= & G_{t}(t) * u_{1}+H_{t}(t) * u_{0} \\
& +\int_{0}^{t} G_{t}(t-\tau) *\left(I-a \Delta+\gamma \Delta^{2}\right)^{-1} \Delta f(u(\tau)) d \tau .
\end{aligned}
$$

Using (2.18)-(2.20), (2.22), Lemma 4.1 and (4.1), for $h \leq s$, we arrive at

$$
\begin{aligned}
\left\|\partial_{x}^{h} \Phi(u)_{t}\right\|_{L^{2}} & \\
\leq & C\left\|\partial_{x}^{h} G_{t}(t) * u_{1}\right\|_{L^{2}}+C\left\|\partial_{x}^{h} H_{t}(t) * u_{0}\right\|_{L^{2}} \\
& +C \int_{0}^{t}\left\|\partial_{x}^{h} G_{t}(t-\tau) *\left(I-a \Delta+\gamma \Delta^{2}\right)^{-1} \Delta f(u(\tau))\right\|_{L^{2}} d \tau \\
\leq & C(1+t)^{-n / 4-h / 2-1}\left(\left\|u_{0}\right\|_{\dot{H}_{1}^{-1}}+\left\|u_{1}\right\|_{\dot{H}_{1}^{-2}}\right)+C e^{-c t}\left(\left\|u_{0}\right\|_{H^{s+2}}+\left\|u_{1}\right\|_{H^{s}}\right) \\
& +C \int_{0}^{t / 2}(1+t-\tau)^{-n / 4-h / 2-1}\|f(u)\|_{L^{1}} d \tau \\
& +C \int_{t / 2}^{t}(1+t-\tau)^{-n / 4-1}\left\|\partial_{x}^{h} f(u)\right\|_{L^{1}} d \tau+C \int_{0}^{t} e^{-c(t-\tau)}\left\|\partial_{x}^{h} f(u)\right\|_{L^{2}} d \tau \\
\leq & C(1+t)^{-n / 4-h / 2-1}\left(\left\|u_{0}\right\|_{\dot{H}_{1}^{-1}}+\left\|u_{1}\right\|_{\dot{H}_{1}^{-2}}\right)+C e^{-c t}\left(\left\|u_{0}\right\|_{H^{s+2}}+\left\|u_{1}\right\|_{H^{s}}\right) \\
& +C \int_{0}^{t / 2}(1+t-\tau)^{-n / 4-h / 2-1}\|u\|_{L^{2}}^{2}\|u\|_{L^{\infty}}^{\theta-1} d \tau \\
& +C \int_{t / 2}^{t}(1+t-\tau)^{-n / 4-1}\left\|\partial_{x}^{h} u\right\|_{L^{2}}^{2}\|u\|_{L^{\infty}}^{\theta-1} d \tau+C \int_{0}^{t} e^{-c(t-\tau)}\left\|\partial_{x}^{h} u\right\|_{L^{2}}\|u\|_{L^{\infty}}^{\theta} d \tau
\end{aligned}
$$




$$
\begin{aligned}
\leq & C(1+t)^{-n / 4-h / 2-1}\left(\left\|u_{0}\right\|_{\dot{H}_{1}^{-1}}+\left\|u_{1}\right\|_{\dot{H}_{1}^{-2}}\right)+C e^{-c t}\left(\left\|u_{0}\right\|_{H^{s+2}}+\left\|u_{1}\right\|_{H^{s}}\right) \\
& +C R^{\theta+1} \int_{0}^{t / 2}(1+t-\tau)^{-n / 4-h / 2-1}(1+\tau)^{-(n / 2+1)}(1+\tau)^{-(n / 2+1 / 2)(\theta-1)} d \tau \\
& +C R^{\theta+1} \int_{t / 2}^{t}(1+t-\tau)^{-n / 4-1}(1+\tau)^{-n / 2-h-1}(1+\tau)^{-(n / 2+1 / 2)(\theta-1)} d \tau \\
& +C R^{\theta+1} \int_{0}^{t} e^{-c(t-\tau)}(1+\tau)^{-n / 4-h / 2-1}(1+\tau)^{-(n / 2+1 / 2) \theta} d \tau \\
\leq & C(1+t)^{-n / 4-h / 2-1}\left\{\left(\left\|u_{0}\right\|_{\dot{H}_{1}^{-1}}+\left\|u_{1}\right\|_{\dot{H}_{1}^{-2}}+\left\|u_{0}\right\|_{H^{s+2}}+\left\|u_{1}\right\|_{H^{s}}\right)+R^{\theta+1}\right\} .
\end{aligned}
$$

Thus

$$
(1+t)^{n / 4+h / 2+1}\left\|\partial_{x}^{h} \Phi(u)_{t}\right\|_{L^{2}} \leq C E_{0}+C R^{\theta+1} .
$$

Taking $E_{0}$ and $R$ suitably small and combining (4.5) and (4.7), we get

$$
\|\Phi(u)\|_{X} \leq R .
$$

For $u, v \in X_{R}$, by using (4.4), we obtain

$$
\Phi(u)-\Phi(v)=\int_{0}^{t} G(t-\tau) *\left(I-a \Delta+\gamma \Delta^{2}\right)^{-1} \Delta[f(u)-f(v)] d \tau .
$$

Using (4.9), (2.21) and Lemma 4.2 and (4.1), for $k \leq s+2$, we obtain

$$
\begin{aligned}
\left.\| \partial_{x}^{k} \Phi(u)-\Phi(v)\right) \|_{L^{2}} & \int_{0}^{t}\left\|\partial_{x}^{k} G(t-\tau) *\left(I-a \Delta+\gamma \Delta^{2}\right)^{-1} \Delta[f(u)-f(v)]\right\|_{L^{2}} d \tau \\
\leq & C \int_{0}^{t / 2}(1+t-\tau)^{-n / 4-k / 2-1 / 2}\|[f(u)-f(v)]\|_{L^{1}} d \tau \\
& +C \int_{t / 2}^{t}(1+t-\tau)^{-n / 4-1 / 2}\left\|\partial_{x}^{k}[f(u)-f(v)]\right\|_{L^{1}} d \tau \\
& +C \int_{0}^{t} e^{-c(t-\tau)}\left\|\partial_{x}^{k}[f(u)-f(v)]\right\|_{L^{2}} d \tau \\
\leq & C \int_{0}^{t / 2}(1+t-\tau)^{-n / 4-k / 2-1 / 2}\left(\|\tilde{u}\|_{L^{2}}+\|\bar{u}\|_{L^{2}}\right)\|u-v\|_{L^{2}} \\
& \times\left(\|u\|_{L^{\infty}}+\|v\|_{L^{\infty}}\right)^{\theta-1} d \tau \\
& +C \int_{t / 2}^{t}(1+t-\tau)^{-n / 4-1 / 2}\left\{\left(\left\|\partial_{x}^{k} u\right\|_{L^{2}}+\left\|\partial_{x}^{k} u\right\|_{L^{2}}\right)\|u-v\|_{L^{2}}\right. \\
& \left.+\left(\|u\|_{L^{2}}+\|v\|_{L^{2}}\right)\left\|\partial_{x}^{k}(u-v)\right\|_{L^{2}}\right\}\left(\|u\|_{L^{\infty}}+\|v\|_{L^{\infty}}\right)^{\theta-1} d \tau
\end{aligned}
$$




$$
\begin{aligned}
& +C \int_{0}^{t} e^{-c(t-\tau)}\left\{\left(\left\|\partial_{x}^{k} u\right\|_{L^{2}}+\left\|\partial_{x}^{k} u\right\|_{L^{2}}\right)\|u-v\|_{L^{\infty}}\right. \\
& \left.+\left(\|u\|_{L^{\infty}}+\|v\|_{L^{\infty}}\right)\left\|\partial_{x}^{k}(u-v)\right\|_{L^{2}}\right\}\left(\|u\|_{L^{\infty}}+\|v\|_{L^{\infty}}\right)^{\theta-1} d \tau \\
\leq & C R^{\theta}\|u-v\|_{X} \int_{0}^{t / 2}(1+t-\tau)^{-n / 4-k / 2-1 / 2}(1+\tau)^{-(n / 2+1 / 2) \theta} d \tau \\
& +C R^{\theta}\|u-v\|_{X} \int_{t / 2}^{t}(1+t-\tau)^{-n / 4-1 / 2}(1+\tau)^{-((\theta / 2)(n+1)+(k+1) / 2)} d \tau \\
& +C C R^{\theta}\|\tilde{u}-\bar{u}\|_{X} \int_{0}^{t} e^{-c(t-\tau)}(1+\tau)^{-(n / 4+(n / 2) \theta+k / 2+1 / 2)} d \tau \\
\leq & C R^{\theta}(1+t)^{-n / 4-k / 2-1 / 2}\|\tilde{u}-\bar{u}\|_{X},
\end{aligned}
$$

which implies

$$
(1+t)^{n / 4+k / 2+1 / 2}\left\|\partial_{x}^{k}(\Phi(u)-\Phi(v))\right\|_{L^{2}} \leq C R^{\theta}\|u-v\|_{X} .
$$

Similarly for $h \leq s$, from (4.6), (2.22) and (4.1), we have

$$
\begin{aligned}
\left\|\partial_{x}^{h}(\Phi(u)-\Phi(v))_{t}\right\|_{L^{2}} \leq & \int_{0}^{t}\left\|\partial_{x}^{h} G_{t}(t-\tau) *\left(I-a \Delta+\gamma \Delta^{2}\right)^{-1} \Delta[f(u)-f(v)]\right\|_{L^{2}} d \tau \\
\leq & C \int_{0}^{t / 2}(1+t-\tau)^{-n / 4-h / 2-1}\|(f(u)-f(v))\|_{L^{1}} d \tau \\
& +C \int_{t / 2}^{t}(1+t-\tau)^{-n / 4-1}\left\|\partial_{x}^{h}(f(u)-f(v))\right\|_{L^{1}} d \tau \\
& +C \int_{0}^{t} e^{-c(t-\tau)}\left\|\partial_{x}^{h}(f(u)-f(v))\right\|_{L^{2}} d \tau \\
\leq & C R^{\theta}(1+t)^{-n / 4-h / 2-1}\|u-v\|_{X},
\end{aligned}
$$

which implies

$$
(1+t)^{n / 4+h / 2+1}\left\|\partial_{x}^{h}(\Phi(u)-\Psi(v))_{t}\right\|_{L^{2}} \leq C R^{\theta}\|u-v\|_{X} .
$$

Taking $R$ suitably small and using (4.10), (4.11), we obtain

$$
\|\Phi(u)-\Phi(v)\|_{X} \leq \frac{1}{2}\|u-v\|_{X} .
$$

It follows (4.8) and (4.12) that $\Phi$ is strictly contracting mapping. Consequently, we conclude that there exists a fixed point $u \in X_{R}$ of the mapping $\Phi$, which is a classical solution to IVP (1.1) and (1.2). This completes the proof.

Acknowledgement. This work was supported by Hunan Provincial Natural Science Foundation of China No. 2016JJ2061, Scientific Research Fund of Hunan 
Provincial Education Department No. 15B102, China Postdoctoral Science Foundation No. 2013M532169, No. 2014T70991, NNSF of China Grant No. 11371367, NNSF of China Grant No. 11271118, the construct program of the key discipline in Hunan province No. 201176 and Science and technology innovation team in Colleges and universities in Hunan Province.

\section{REFERENCES}

[1] J. BoussinesQ, Théorie des ondes et des remous qui se propagent le long dun canal rectangulaire horizontal en communiquant au liquide contenu dans ce canal des vitesses sensiblement pareilles de la surface au fond, J. Math. Pures Appl. 17 (1872), 55-108.

[2] Y. Cho AND T. Ozawa, Remarks on modified improved Boussinesq equations in one space dimension, Proc. R. Soc. Lond. Ser. A. Math. Phys. Eng. Sci. 462 (2006), 1949-1963.

[ 3 ] Y. Cho And T. Ozawa, On small amplitude solutions to the generalized Boussinesq equations, Discrete Contin. Dyn. Syst. 17 (2007), 691-711.

[ 4 ] G. Chen AND X. HAN, Global existence of solution of Cauchy problem for a nonlinear wave equation, IMA J. Appl. Math. 78 (2013), 1015-1031.

[ 5] A. DÉ Godefroy, Blow up of solutions of a generalized Boussinesq equation, IMA J. Appl. Math. 60 (1998), 123-138.

[6] S. LAI AND Y. WU, The asymptotic solution of the Cauchy problem for a generalized Boussinesq equation, Discrete Contin. Dyn. Syst. Ser. B. 3 (2003), 401-408.

[ 7] S. LAI, Y. WANG, Y. WU AND Q. LIN, An initial-boundary value problem for a generalized Boussinesq water system in a ball, Int. J. Appl. Math. Sci. 3 (2006), 117-133.

[ 8 ] V. G. Makhankov, Dynamics of classical solitons (in nonintegrablesystems), Physics Reports A review section of Phys. Lett. (Section C) 35 (1978), 1-128.

[9] N. Polat and A. Ertaş, Existence and blow-up of solution of Cauchy problem for the generalized damped multidimensional Boussinesq equation, J. Math. Anal. Appl. 349 (2009), $10-20$.

[10] N. Polat And E. PişKin, Asymptotic behavior of a solution of the Cauchy problem for the generalized damped multidimensional Boussinesq equation, Appl. Math. Lett. 25 (2012), 1871-1874.

[11] E. PişKIn AND N. Polat, Existence, global nonexistence, and asymptotic behavior of solutions for the Cauchy problem of a multidimensional generalized damped Boussinesq-type equation, Turk. J. Math. 38 (2014), 706-727.

[12] J. Shen, Y. YANG AND R. XU, Global existence of solutions for 1-D nonlinear wave equation of sixth order at high initial energy level, Boundary Value Problems 31 (2014), 1-6.

[13] V. Varlamov, Existence and uniqueness of a solution to the Cauchy problem for the damped Boussinesq equation, Math. Methods Appl. Sci. 19 (1996), 639-649.

[14] V. Varlamov, On the initial-boundary value problem for the damped Boussinesq equation, Discrete Contin. Dyn. Syst. 4 (1998), 431-444.

[15] V. Varlamov, Eigenfunction expansion method and the long-time asymptotics for the damped Boussinesq equation, Discrete Contin. Dyn. Syst. 7 (2001), 675-702.

[16] V. Varlamov, On the spatially two-dimensional Boussinesq equation in a circular domain, Nonlinear Anal. T.M.A. 46 (2001), 699-725.

[17] V. Varlamov and A. Balogh, Forced nonlinear oscillations of elastic membranes, Nonlinear Anal. R.W. A. 7 (2006), 1005-1028.

[18] S. WANG AND G. ChEN, The Cauchy problem for the generalized IMBQ equation in $W^{s, p}\left(\mathbf{R}^{n}\right)$, J. Math. Anal. Appl. 274 (2002), 38-54. 
[19] S. Wang And H. Xue, Global solution for a generalized Boussinesq equation, Appl. Math. Comput. 204 (2008), 130-136.

[20] S. Wang AND M. LI, The Cauchy problem for coupled IMBq equations, IMA J. Appl. Math. 74 (2009), 726-740.

[21] Y. WANG, C. Mu AND Y. WU, Decay and scattering of solutions for a generalized Boussinesq equation, J. Differential Equations 247 (2009), 2380-2394.

[22] B. X. Wang, Z. H. Huo, C. C. Chao and Z. H. Guo, Harmonic analysis method for nonlinear evolution equations, World Scientific Press, New York, 2011.

[23] S. WANG AND G. ChEN, Small amplitude solutions of the generalized IMBQ equation, J. Math. Anal. Appl. 274 (2002), 846-866.

[24] S. WANG AND H. XU, On the asymptotic behavior of solution for the generalized IBq equation with hydrodynamical damped term, J. Differential Equations 252 (2012), 4243-4258.

[25] S. Wang AND F. DA, On the asymptotic behavior of solution for the generalized double dipersion equation, Appl. Anal. 92 (2013), 1179-1193.

[26] S. XIA AND J. YUAN, Existence and scattering of small solutions to a Boussinesq type equation of sixth order, Nonlinear Anal. 73 (2010), 1015-1027.

[27] S. M. Zheng, Nonlinear evolution equations, Monographs and Surveys in Pure and Applied Mathematics, 133, Chapan Hall/CRC, New York, 2004.

[28] Y. Zhang, Q. Lin and S. LaI, Long time asymptotic for the damped Boussinesq equation in a circle, J. Partial Diff. Eqs. 18 (2005), 97-113.

\author{
Zaiyun Zhang \\ SchoOl of Mathematics \\ Hunan Institute of Science and Technology \\ YUEYANG 414006, HuNAN \\ P. R. CHINA \\ College of Science \\ National University of Defense Technology \\ Changsha 410073, Hunan \\ P. R. CHINA \\ E-mail: zhangzaiyun1226@126.com \\ Jianhua Huang \\ College of SCIENCE \\ National University of Defense Technology \\ Changsha 410073, Hunan \\ P. R. China \\ E-mail: jhhuang32@nudt.edu.cn \\ Mingbao Sun \\ SchoOl of Mathematics \\ Hunan Institute of Science and Technology \\ YUEYANG 414006, HUNAN \\ P. R. CHINA \\ E-mail: sun_mingbao@163.com
}

